\title{
Relentless Placoid Chorioretinitis: A Case Series of Successful Tapering of Systemic Immunosuppressants Achieved with Adalimumab
}

\author{
Shotaro Asano ${ }^{a}$ Rie Tanaka ${ }^{a} \quad H^{2}$ idetoshi Kawashima ${ }^{b}$ \\ Toshikatsu Kaburaki ${ }^{\mathrm{a}}$ \\ aDepartment of Ophthalmology, University of Tokyo Graduate School of Medicine, \\ Tokyo, Japan; 'bepartment of Ophthalmology, Jichi Medical University, Tochigi, Japan
}

\section{Keywords}

Relentless placoid chorioretinitis · Adalimumab - Uveitis - Acute posterior multifocal placoid pigment epitheliopathy · Serpiginous choroiditis

\begin{abstract}
Background: Adalimumab, a human anti-tumor necrosis factor-a monoclonal antibody, was recently reported to be effective in lowering the risk of recurrence of noninfectious uveitis. This is the first case series of adalimumab administrations for relentless placoid chorioretinitis (RPC) patients. Case Presentation: We report 2 cases of RPC where successful treatments were achieved with adalimumab. A 34-year-old woman developed conjunctival hyperemia, mild iridocyclitis, and multiple atrophic retinal lesions, along with exudative changes that were widespread from the posterior pole to peripheral retina in both eyes. The diagnosis of RPC was made based on the characteristic recurrences of choroiditis despite systemic corticosteroid and cyclosporine. Adalimumab therapy was introduced to the patient, and thereafter no recurrence was observed while tapering the immunosuppressive agents. The second case was a 22-year-old man with visual deterioration in both eyes who exhibited widespread multiple chorioretinal atrophic lesions. We diagnosed the case as RPC based on characteristic clinical findings and recurring chorioretinitis during tapering of systemic corticosteroids. Adalimumab therapy was administrated, and immunosuppressant dosage was successfully reduced without
\end{abstract}


any recurrences. Conclusions: In the current two RPC cases, adalimumab was quite effective and useful to reduce the dosages of systemic immunosuppressants. Further study is necessary to confirm the effectiveness of adalimumab in RPC patients.

\section{Introduction}

Relentless placoid chorioretinitis (RPC) is a disease entity reported by Jones et al. [1]. Clinical features include creamy white retinal lesions that resembles those of acute posterior multifocal placoid pigment epitheliopathy (APMPPE) and serpiginous choroiditis [1-3]. RPC patients pose prolonged periods of widespread lesions [4], which may appear and disappear anterior to the equator, developing into numerous ( $>50$ ) atrophic lesions [1-3]. Systemic corticosteroids are usually administered for RPC treatment, with subsequent healing and visual acuity improvement. However, the recurrence is common during systemic corticosteroid tapering [1-3].

Adalimumab, a human anti-tumor necrosis factor-a monoclonal antibody, was recently reported to be effective in reducing the recurrence of noninfectious uveitis and dosage of systemic corticosteroids and immunomodulators [5]. Herein, we present 2 cases of RPC in which adalimumab treatment successfully led to systemic immunosuppressant reduction without any relapses.

\section{Case Presentations}

Case 1

A 34-year-old woman suffered hyperemia and blurred vision in the right eye half a year ago and visited a local clinic. Multifocal choroiditis in the right eye was suspected, and she received topical corticosteroid and subconjunctival injection of triamcinolone. However, she developed visual field deterioration in both eyes and was referred to our clinic. Best-corrected visual acuity (BCVA) was 20/200 (OD) and 20/16 (OS). We detected bilateral conjunctival hyperemia, mild iridocyclitis in the right eye, and multiple atrophic retinal lesions with some exudative changes widespread from the posterior pole to peripheral retina in both eyes (Fig. 1a). Fundus autofluorescence presented hypoautofluorescent regions corresponding to the outer retinal atrophy (Fig. 1b), and fluorescein angiography (FA) demonstrated late staining in the foveal region, especially from the margins of the region and vascular leakage in peripheral region (Fig. 1c). Late hyperfluorescence of the discs in both eyes was also observed. By optical coherence tomography (OCT) in the right eye, subretinal fluid in the subfoveal region and hyperreflective materials in ellipsoid zone were detected. Hyperplasia of retinal pigment epithelium (RPE) and ellipsoid zone were also observed (Fig. 1d). Complete blood count, erythrocyte sedimentation rate, C-reactive protein, kidney function tests, and liver function test were normal, and serology for syphilis and HIV were negative. Normal chest computed tomography, negative purified-protein derivative reaction, and negative T-SPOT.TB excluded the diagnosis of sarcoidosis and tuberculosis. Diagnosis of multifocal choroiditis and uveitis was made. We prescribed oral prednisolone at $30 \mathrm{mg} /$ day and $0.1 \%$ betamethasone eyedrops for the right eye 4 times a day. After retinal exudative changes were alleviated, we began tapering both medications.

One month after the first visit (oral prednisolone: $10 \mathrm{mg} /$ day), chorioretinitis near the disc and peripheral fundus relapsed in the left eye, and we increased oral prednisolone to 25 
$\mathrm{mg}$ /day which improved the condition, and gradual tapering was restarted. Twelve months after the initial visit (oral prednisolone: $7 \mathrm{mg} /$ day), the recurrence with multiple exudative changes in the periphery was observed in both eyes. In consideration of the clinical course and appearance, we made a diagnosis of RPC. We increased oral prednisolone (15 mg/day) and added oral cyclosporine $(200 \mathrm{mg} /$ day). Nevertheless, relapse occurred over the next 5 months. Thus, 17 months after the initial treatment, subcutaneous administration of adalimumab (40 mg/2 weeks) was introduced. Following the introduction, the oral immunosuppressants were drastically tapered without recurrence or any drug complications related to adalimumab. Three months after adalimumab therapy was started, oral cyclosporine was terminated. One year after introduction of adalimumab, oral prednisolone was successfully terminated without recurrence. BCVA had recovered up to 20/16 in both eyes. OCT image demonstrated alleviation of RPE hyperplasia, turned into rarefaction of outer retina and ellipsoid zone (Fig. 1e).

Case 2

A 22-year-old man was examined at a local clinic for visual deterioration in both eyes, and widespread multiple chorioretinal lesions were found in both fundi (Fig. 2a). Fundus autofluorescence demonstrated increase in central hypoautofluorescence within the lesions corresponding to the outer retinal atrophy and RPE hyperplasia (Fig. 2b). FA demonstrated hyperfluorescence of the lesions in late phase (Fig. 2c), and indocyanine green angiography showed hypofluorescence in the area coincide with the clinical legions (Fig. 2d). Similar to the previous case, laboratory work-up, including complete blood count, erythrocyte sedimentation rate, C-reactive protein, kidney function tests, and liver function test were within normal limits, and serology for syphilis and HIV were negative. Normal chest computed tomography, negative purified-protein derivative reaction, and negative T-SPOT.TB. denied sarcoidosis and tuberculosis. Oral prednisolone at $20 \mathrm{mg} /$ day and oral cyclosporine at $300 \mathrm{mg} /$ day were started. One month later, at the initial visit to our hospital, BCVA was 20/60 (OD) and 20/20 (OS). No inflammatory signs were observed. Thus, oral prednisolone prescription was gradually tapered.

Six months later (oral prednisolone: $11 \mathrm{mg} /$ day, oral cyclosporine: $150 \mathrm{mg} /$ day), relapse with new chorioretinal exudative changes occurred in the left eye. A diagnosis of RPC was made and oral prednisolone was increased to $15 \mathrm{mg} /$ day. Once ocular inflammatory changes had subsided, adalimumab ( $40 \mathrm{mg} / 2$ weeks) was started and cyclosporine was discontinued. Since then, no recurrence occurred over an 9-month period, and oral prednisolone was successfully tapered to $5 \mathrm{mg} /$ day. No complications related to the use of adalimumab was noted. BCVA recovered to 20/20 (OD) and 20/16 (OS). Change in OCT findings are shown in Figure 3. Small amount of subretinal hyperreflective materials, focal outer retinal disruption, and subfoveal RPE hyperplasia were observed at the first visit (Fig. 3a). Nine months after the administration of adalimumab, the recession of RPE hyperplasia according to the inactivation of inflammation was detected, albeit partial hyperplasia remained. Ellipsoid zone was rarefied at the site of prior hyperplasia (Fig. 3b).

\section{Discussion}

We report 2 patients with RPC, for whom we were able to reduce systemic immunosuppressant dosage successfully without recurrence probably thanks to adalimumab. RPC is characterized by bilateral widespread distribution of retinal white lesions [1-3]. Multimodal 


\section{Case Reports in Ophthalmology}

images of both cases demonstrated typical clinical findings. In the current cases, numerous multifocal recurrent chorioretinitis and atrophic lesions in more than 50 locations, which appear from posterior pole to peripheral retina, were observed. FA was carried out, and characteristic early hypofluorescence with late staining were observed [1]. OCT images can show hyperplasia of RPE and ellipsoid zone at the active phase, which suggest chorioretinal pathogenetic processes of RPC [6]. All these findings in multimodal images of our cases coincide with the clinical findings of previously reported RPC cases [1-4, 6]. After the inflammation improved, hyperplasia decreased and rarefaction of the outer retina, i.e. ellipsoid zone and RPE, developed. Systemic medical workup, clinical manifestation, and clinical course of those cases excluded diseases such as APMPPE, serpiginous choroiditis, multifocal choroiditis, syphilis, tuberculosis, sarcoidosis, viral-associated uveitis, and uveitis associated with rheumatologic diseases [1].

In eyes with RPC, prolonged clinical course with repeated recurrence often occurs [1-3], hence, the relentless course beyond 6 months is often the key to its diagnosis and differentiation from APMPPE and serpiginous choroiditis [4]. The current cases both demonstrated the prolonged clinical periods of more than 6 months, then the clinical diagnoses were confirmed. Because of the high relapse and the risk of continuing systemic corticosteroid administration, tapering of immunosuppressants and corticosteroid needs to be done carefully in RPC management [1]. One previous case report suggested the usefulness of intravitreal triamcinolone acetonide (IVTA) for RPC in a patient for whom systemic corticosteroid administration was considered to be risky because of pregnancy [7]. However, a posterior subcapsular cataract and noninfectious endophthalmitis developed in that patient, which might have been caused by IVTA. In our cases, the patients suffered no adverse effects related to adalimumab and systemic corticosteroid and immunosuppressant were successful tapered without recurrence.

After adalimumab was introduced, the disease activity started to diminish, and systemic corticosteroid was tapered without any relapses. Thus, our cases imply an effectiveness of adalimumab in reducing systemic corticosteroids drastically. The previous studies also indicated the usefulness of adalimumab in reducing systemic corticosteroid and immunosuppressant dosage in recurrent noninfectious uveitis management [5]. Both of the cases were still treated with adalimumab at the time of the final visit. Considering adalimumab's role in preventing recurrences, the patient needs to be monitored closely with respect to tapering or terminating adalimumab. The current report was only 2 cases, and the standard therapy of RPC has yet to be determined [1]; hence, further studies with larger number of patients are warranted to confirm the effectiveness of adalimumab in RPC patients.

\section{Conclusion}

To the best of our knowledge, this is the first report documenting the effectiveness of adalimumab for RPC. Although only 2 cases are presented, adalimumab may have the potential to effectively suppress recurrence associated with RPC. Additional examinations and case reports are critical for a better understanding of this issue.

\section{Statement of Ethics}

The study was approved by the Institutional Review Board of the University of Tokyo and adhered to the tenets of the Declaration of Helsinki. Written informed consent was obtained 
from the patients for participation. Written informed consent was obtained from the patients for publication.

\section{Disclosure Statement}

The authors have no conflicts of interest to declare.

\section{Funding Sources}

This work was supported by a Grant-in-Aid for Scientific Research from the Japan Society for the Promotion of Science (KAKENHI Grant No. 18K09398), as well as a grant for Research on Specific Diseases of the Health Sciences Research from the Ministry of Health, Labour and Welfare (H29-nanti-ippan-050), and grants from Eisai Japan and Novartis Pharma.

\section{Author Contributions}

S.A. analyzed the patient data regarding the relationship between relentless placoid chorioretinitis and use of adalimumab and systemic immunosuppressants, and was a major contributor in writing the manuscript. R.T., H.K., and T.K. interpreted the medication dosage and activity of the disease. All authors read and approved the final manuscript.

\section{References}

1 Jones BE, Jampol LM, Yannuzzi LA, Tittl M, Johnson MW, Han DP, et al. Relentless placoid chorioretinitis: A new entity or an unusual variant of serpiginous chorioretinitis? Arch Ophthalmol. 2000 Jul;118(7):931-8.

2 Dolz-Marco R, Rodríguez-Ratón A, Hernández-Martínez P, Pascual-Camps I, Andreu-Fenoll M, Gallego-Pinazo R. Macular retinal and choroidal thickness in unilateral relentless placoid chorioretinitis analyzed by sweptsource optical coherence tomography. J Ophthalmic Inflamm Infect. 2014 Oct;4(1):24.

3 Mirza RG, Jampol LM. Relentless placoid chorioretinitis. Int Ophthalmol Clin. 2012;52(4):237-42.

4 Raven ML, Ringeisen AL, Yonekawa Y, Stem MS, Faia LJ, Gottlieb JL. Multi-modal imaging and anatomic classification of the white dot syndromes. Int J Retina Vitreous. 2017 Mar;3(1):12.

5 Suhler EB, Thorne JE, Mittal M, Betts KA, Tari S, Camez A, et al. Corticosteroid-Related Adverse Events Systematically Increase with Corticosteroid Dose in Noninfectious Intermediate, Posterior, or Panuveitis: Post Hoc Analyses from the VISUAL-1 and VISUAL-2 Trials. Ophthalmology. 2017 Dec;124(12):1799-807.

6 Amer R, Florescu T. Optical coherence tomography in relentless placoid chorioretinitis. Clin Exp Ophthalmol. 2008 May;36(4):388-90.

7 Roth DB, Ballintine S, Mantopoulos D, Prenner J, Fine HF. Relentless Placoid Chorioretinitis: Successful LongTerm Treatment with Intravitreal Triamcinolone. Retin Cases Brief Rep. 2019 Spring;13(2):150-153. 


\section{Case Reports in Ophthalmology}
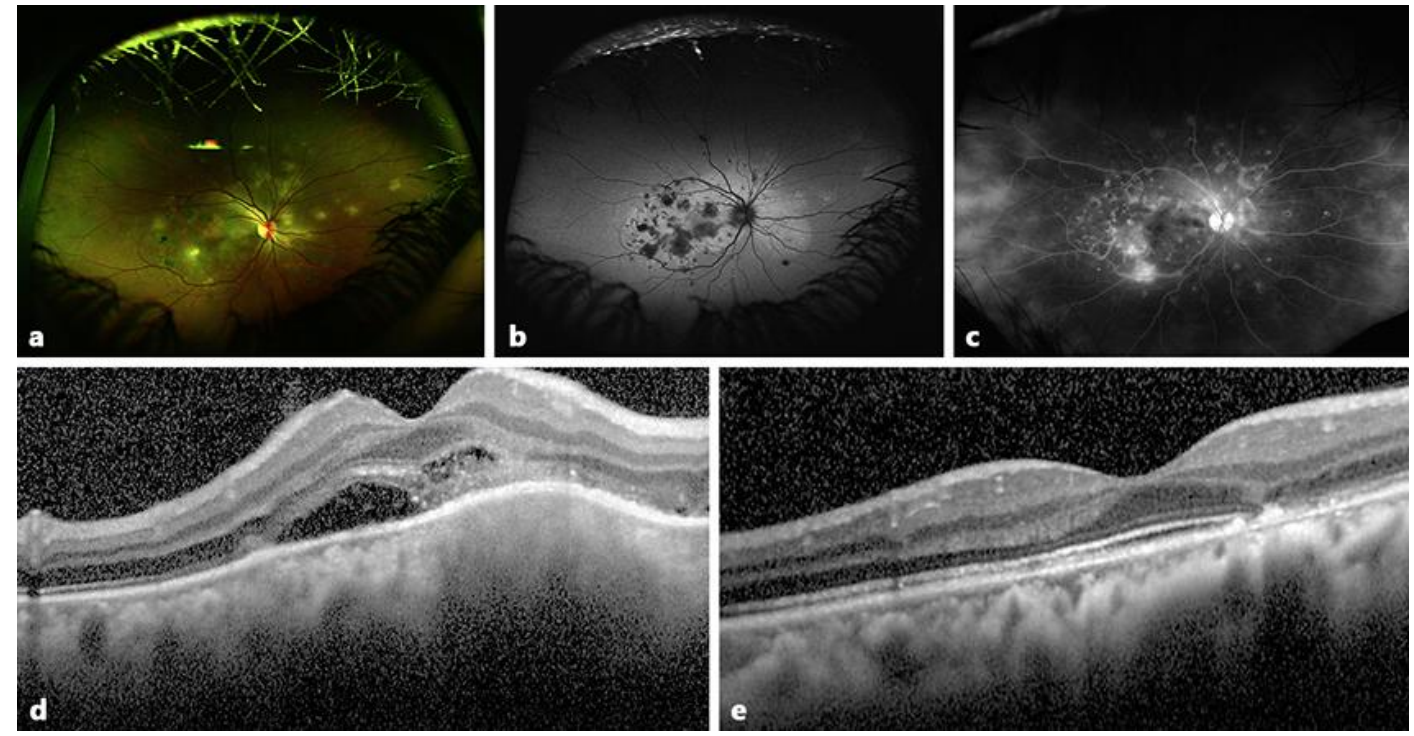

Fig. 1. Fundus photographs of case 1. a Multiple atrophic retinal lesions with ultrawide-field color fundus photograph. b Fundus autofluorescence images. c Late-phase hyperfluorescence of the lesions with fluorescein angiography. $\mathbf{d}$ Subretinal fluid in the subfoveal region, hyperreflective materials in ellipsoid zone, and hyperplasia of retinal pigment epithelium (RPE) and ellipsoid zone presented with optical coherence tomography (OCT) image at the administration. e OCT images 1 year after introduction of adalimumab demonstrated alleviation of RPE hyperplasia. 


\section{Case Reports in Ophthalmology}
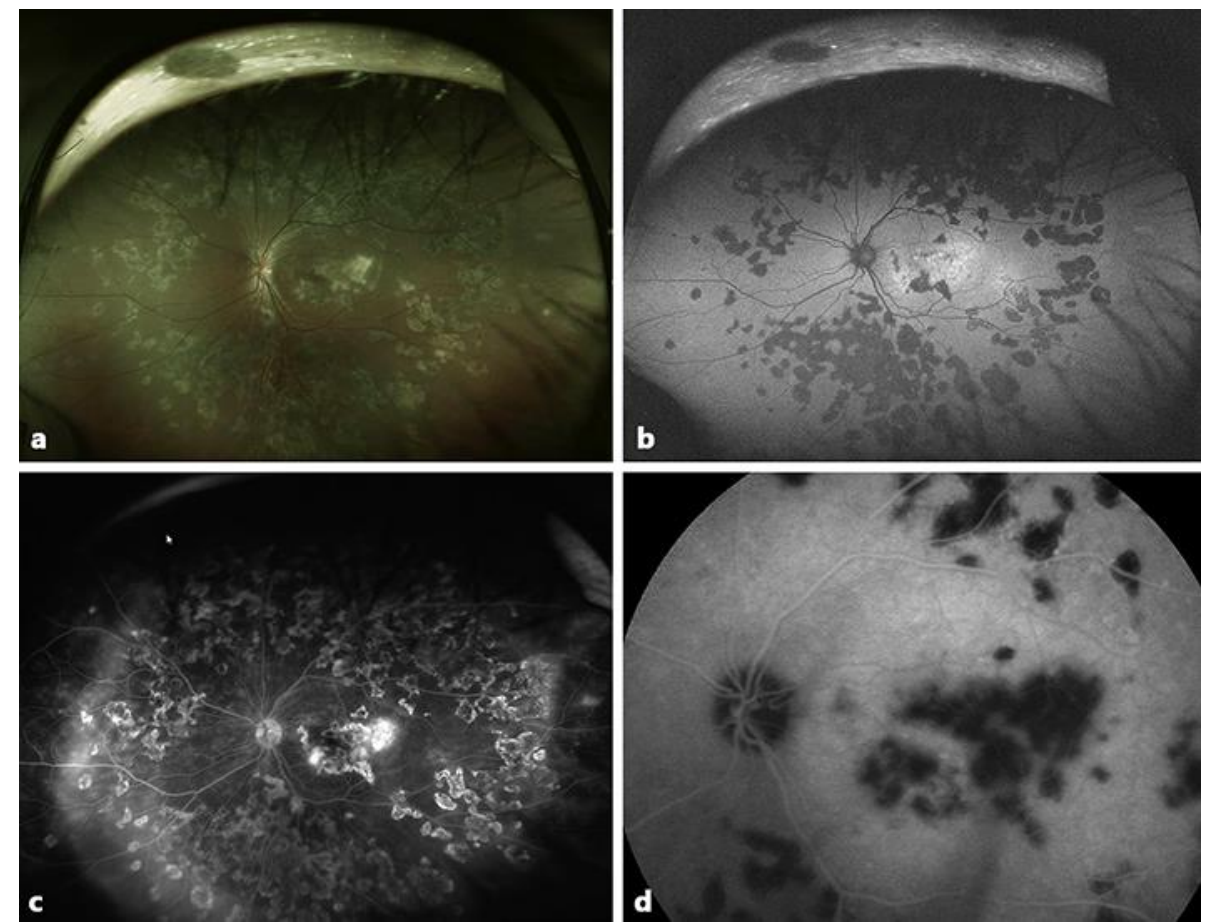

Fig. 2. Fundus photographs of case 2. a Ultrawide-field color fundus photograph shows widespread multiple chorioretinal atrophic lesions. b Central hypoautofluorescence demonstrated with fundus autofluorescence image. $\mathbf{c}$ Late-phase hyperfluorescence of the lesions with fluorescein angiography. $\mathbf{d}$ Late-phase hyperfluorescence in the area coincides with the clinical lesions with indocyanine green angiography. 


\section{Case Reports in Ophthalmology}

\begin{tabular}{l|l}
\hline Case Rep Ophthalmol 2019;10:145-152 \\
\hline DOI: 10.1159/000500077 & $\begin{array}{l}\text { @ } 2019 \text { The Author(s). Published by S. Karger AG, Basel } \\
\text { www.karger.com/cop }\end{array}$ \\
\hline
\end{tabular}

Asano et al.: Applying Adalimumab in Relentless Placoid Chorioretinitis
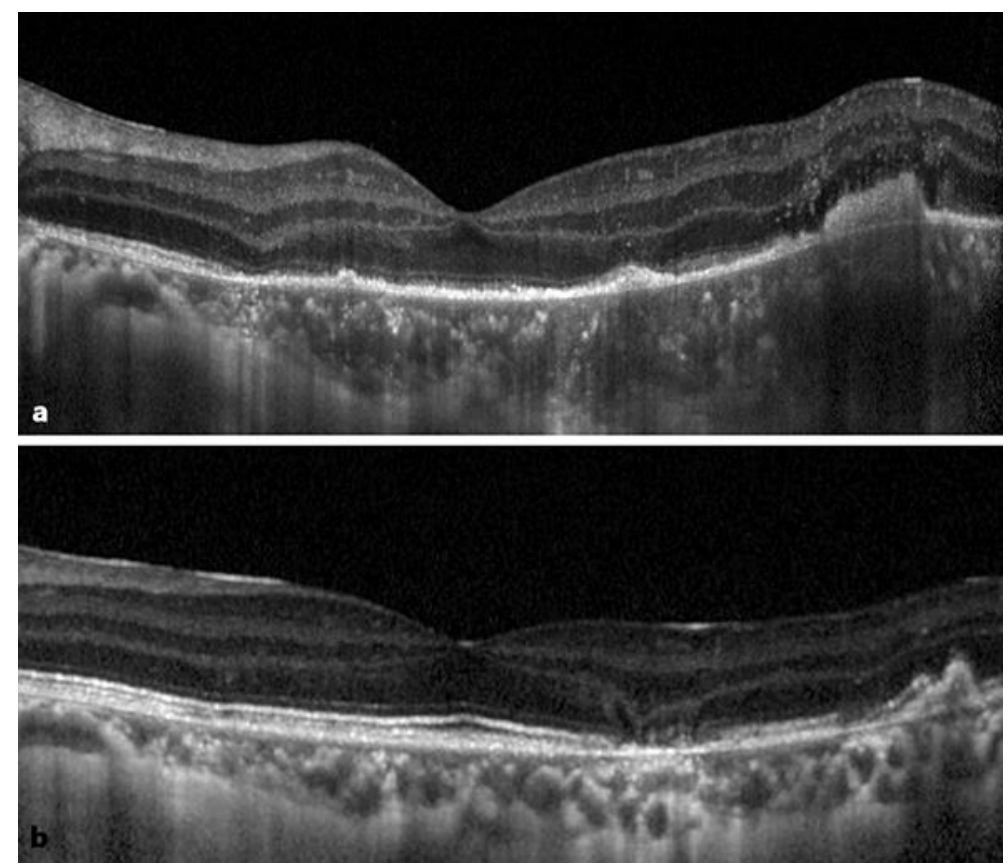

Fig. 3. Subfoveal optical coherence tomography images of case 2. a Small amount of subretinal hyperreflective materials, focal outer retinal disruption, and subfoveal retinal pigment epithelium (RPE) hyperplasia were observed at the administration. $\mathbf{b}$ The recession of RPE hyperplasia occurred 9 months after the introduction of adalimumab. 